\title{
Radiographic features of the dorsal condylar sagittal ridge of the third metacarpal and metatarsal bones in young Warmblood stallions
}

\author{
S. Hauspie1; A. Martens²; J. Declercq²; V. Busoni³; K. Vanderperren'; H. van Bree"; \\ J. H. Saunders ${ }^{1}$ \\ 'Department of Veterinary Medical Imaging and Small Animal Orthopaedics, Faculty of Veterinary Medicine, Ghent \\ University, Salisburylaan, Belgium; ${ }^{2}$ Department of Surgery and Anaesthesia of Domestic Animals, Faculty of Veterin- \\ ary Medicine, Ghent University, Salisburylaan, Belgium; ${ }^{3}$ Department of Medical Imaging, Faculty of Veterinary Medi- \\ cine, University of Liege, Sart-Tilman; Belgium
}

\section{Keywords}

Equine, metacarpophalangeal joint, metatarsophalangeal joint, radiography, Warmblood stallion

\section{Summary}

Objectives: To describe the radiographic appearance of the dorsoproximal aspect of the sagittal ridge of the third metacarpal/metatarsal bone in Warmblood horses.

Methods: The lateromedial radiographic projections of the metacarpo-/metatarsophalangeal joints performed on horses as a part of stallion selection were used. The dorsal aspect of the distal third metacarpal/metatarsal bone was divided in two areas. The appearance of the bone surface in area I was classified as normal, irregular, notch, indentation and lucency. For area II, the categories were normal, irregular, depression or lucency and flattening of the sagittal ridge. Other abnormalities at the dorsal aspect were also noted.
Results: In area I, $51.5 \%$ of the ridges appeared normal, $19.3 \%$ were irregular, $8.9 \%$ had a notch, $8.1 \%$ had a lucency, and $12.2 \%$ had an indentation. In $1.2 \%$ of the horses a fragment was present, and in $1.7 \%$ a fragment was suspected.

In area II, $90.6 \%$ of the metacarpo-/metatarsophalangeal joints were normal, $6.2 \%$ were irregular, $2.9 \%$ showed a depression or lucen$c y$, and the sagittal ridge in $0.2 \%$ was flattened. A fragment was present in $0.3 \%$, and suspected in $0.4 \%$.

Clinical significance: Morphological variation is present at the dorsal aspect of the metacarpo-/metatarsophalangeal joint in young Warmblood stallions. These various aspects should be recognised and described in horses presented for prepurchase examination. However, their clinical relevance in the individual horse is unclear and needs further investigation.
Correspondence to:

Stijn Hauspie, DVM

Faculty of Veterinary Medicine

Ghent University

Department of Veterinary Medical Imaging and Small

Animal Orthopaedics

Salisburylaan 133

9820 Merelbeke

Belgium

Phone: +32 092647640

Fax: +32 092647657

E-mail: Stijn.hauspie@ugent.be

Funding: Stijn Hauspie is a research fellow of the

"Special Research Fund", Ghent University (Belgium).

\section{Introduction}

The dorso-proximal aspect of the equine metacarpo-/metatarsophalangeal joint is composed of a thick joint capsule, including a synovial pad, a layer of cartilage, subchondral bone, a synovial membrane and synovial fluid (1-3). Although the exact function of the synovial pad has not been studied, its location and structure suggest that it acts as a contact interface or cushion between the proximal dorsal rim of the proximal phalanx and the dorsal surface of the distal third metacarpal/metatarsal bone (MCIII/MTIII) during full extension of the fetlock joint (4). This anatomical region can be affected by specific disorders (e. g. osteochondrosis or chronic proliferative synovitis in the metacarpophalangeal joint), or it can be involved in a generalised joint disorder (e. g. capsulitis or synovitis, osteoarthritis, infectious or traumatic arthritis) $(5,6)$.

An examination is frequently performed prior to the sale of a horse in order to assess the suitability of the animal for the purpose for which it is required. Depending on the intended use and value of the animal, a radiographic examination may be part of the examination. If a decision is made to take radiographic images, then projections of the metacarpo-/metatarso-phalangeal joints will be included (7). Should the veterinarian make a mistake in interpreting the radiographic images, the economic and legal consequences may be disastrous (7). However, there is a general lack of published information regarding the clinical significance of many radiographic findings, as well as the acceptable anatomical variations 


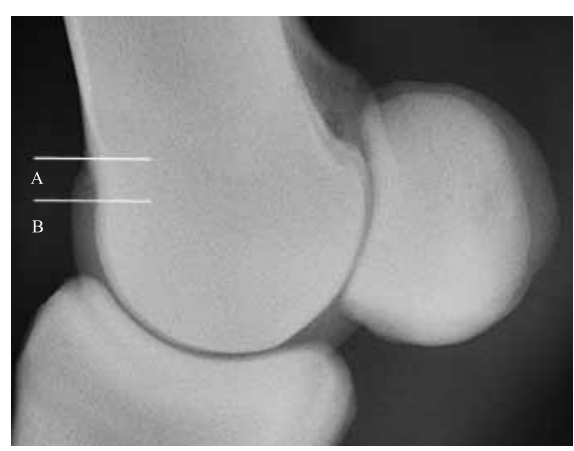

Fig. 1 Lateromedial radiograph of the fetlock. Two regions were used to categorise the radiographic changes on the distal third metacarpal/ metatarsal bone: A) Area I: proximal third of the visible dorsal aspect of the sagittal ridge, at the level of the synovial pad. B) Area II: distal twothirds of the visible dorsal aspect of the sagittal ridge, the region distal to the synovial pad and not superimposed to the proximal phalanx on the lateromedial projection.

(8). Moreover, in addition to the horse's function, the owner's expectations will largely determine their significance (9-11). Because of the difference in type and duration of their sporting careers, great caution should be exercised when using the conclusions drawn from Thoroughbred horses to interpret the radiographs of a Warmblood horse (7-9, 12, 13).

The goal of this study was to describe the radiographic variations observed at the dorso-proximal aspect of the sagittal ridge of the MCIII/MTIII and to assess their relative prevalence in a population of young Warmblood stallions.

\section{Materials and methods}

The latero-medial radiographic projections of the metacarpo- and metatarso-phalangeal joints performed on horses presented at our Institution (Ghent University's Large Animal Teaching Hospital) as a part of stallion selection between April 2007 and March 2009 were used. Only horses younger than six years were used in this study. A short lameness examination was performed: horses were evaluated trotting in a straight line and lunging (hard and soft surface). However, due to the nature of the stallion selection, an in-depth lameness examination, with flexion tests, was not perform-

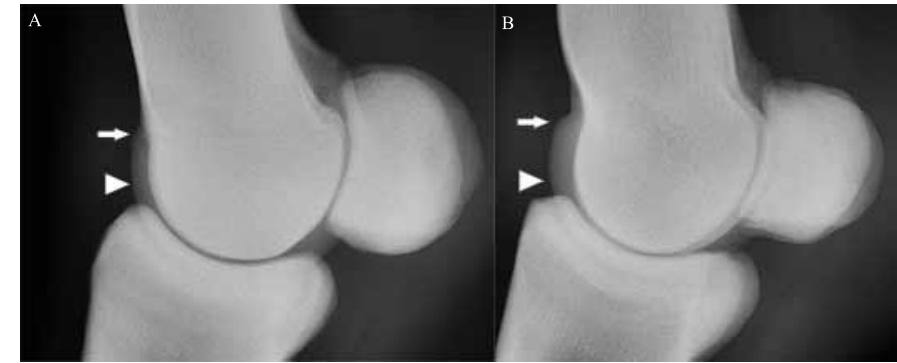

Fig. 2 Lateromedial radiograph of the fetlock showing the difference in shape of the proximal aspect of the sagittal ridge (area I): A) flat and smoothly delineated; B) sharp and smoothly delineated (arrow). The smooth, curved aspect of the distal part of the ridge (area II) is also demonstrated (arrowhead).

ed. Using a computed radiography imaging system, radiographs were made with a horizontal radiographic beam and with the horse bearing weight. The radiographs were evaluated using commercially available software. If the projection was excessively oblique - defined as superimposition of the distal condyles of the MCIII/MTIII on the sagittal ridge, and thus preventing a thorough radiographic interpretation - the radiograph was not used in the study.

Two readers - a board-certified radiologist (JHS) and a $\mathrm{PhD}$-student $(\mathrm{SH})$ - reviewed all of the examinations together, and each decision was made consensually. Special attention was given to the dorsal aspect of the distal MCIII/MTIII, which was divided into two areas ( $>$ Fig. 1).

The first area (area I) corresponded to the proximal third of the visible dorsal aspect of the sagittal ridge, at the level of the synovial pad. The appearance of this area was classified according to the following five categories: normal (defined as flat or sharp and smoothly delineated; $>$ Fig. 2), irregular, well-defined 'notch', irregularly shaped 'lucencies', or a sharply delineated 'indentation' ( Fig. 3) $(13,14)$. The presence of a fragment or a suspected fragment was also noted; if possible, the surface area of the fragment was measured directly from the latero-medial radiographic projection without allowance for magnification. A crude indication of the surface area was obtained by drawing a closed polygon around the outer edges of the fragment and then calculating the surface area with commercially available imaging software ${ }^{\mathrm{a}}$.

OsiriX ${ }^{\circledR}:$ http://www.osirix-viewer.com/
The second area (area II) included the distal two-thirds of the visible dorsal aspect of the sagittal ridge, which corresponds to the region we assumed to be distal to the synovial pad and not superimposed onto the proximal phalanx on the latero-medial projection. For this area, the shape of the sagittal ridge was recorded as: normal (smooth, curved ridge) ( Fig. 2), irregular, showing a depression or lucency, or flattened ( Fig. 4). The presence or suspicion of a fragment, as well as its characteristics, was also recorded. If the fragment was close to (less than $1 \mathrm{~mm}$ ) the dorso-proximal aspect of the proximal phalanx, the fragment was considered to be a fragment at the dorso-proximal aspect of the proximal phalanx and, therefore, it was scored like other radiographic abnormalities (13-15). Statistical analysis was performed to determine if there was a difference between left and right metacarpo-/metatarso-phalangeal joints and between the fore- and hindlimbs. Statistical analysis was performed using the chi-square test (or the Fisher's exact test if sample sizes were small).

\section{Results}

\section{Animals}

A total of 1232 radiographs of metacarpo-/ metatarso-phalangeal joints of 308 stallions were available for this retrospective evaluation. The mean age of the population was 2.75 years ( Fig. 5). None of the horses showed lameness. Twenty-eight radiographs were excluded from the study because of excessive obliquity. In total, 1204 
Fig. 3

Lateromedial radiograph of the fetlock showing changes recorded at the proximal aspect of the sagittal ridge (area I): A) irregular; B) notch; C) lucency; D) indentation.
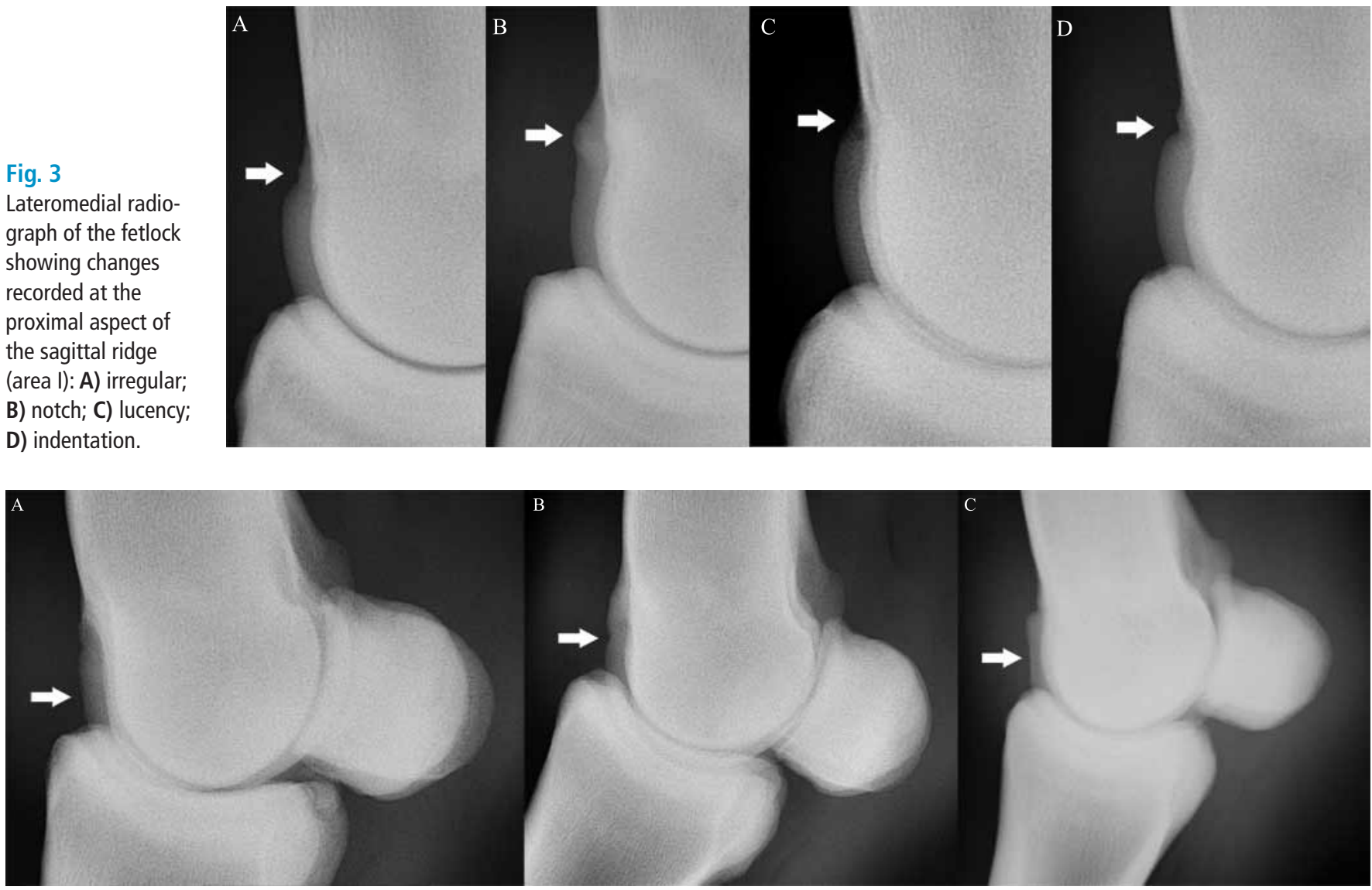

Fig. 4 Lateromedial radiograph of the fetlock showing changes recorded at the distal aspect of the sagittal ridge (area II): A) irregular; B) depression or lucency; C) flattened.

radiographs were used for interpretation: 594 were of forelimbs and 610 were of hindlimbs.

\section{Radiographic findings in area I}

The radiographic findings are summarised in $>$ Table 1 . In area I, $51.5 \%(\mathrm{n}=$ 620 ) of the distal MCIII/MTIII had a normal appearance, $19.3 \%(\mathrm{n}=232)$ were irregular, $8.9 \%(\mathrm{n}=107)$ had a notch, $8.1 \%$ $(\mathrm{n}=98)$ had a lucent area, and $12.2 \%(\mathrm{n}=$ 147) had a well-defined indentation ( Fig. 3). There were not any significant differences found between left and right metacarpo- and metatarsophalangeal joints. A significant difference $(\mathrm{p}<0.0001)$ was found between forelimbs and hindlimbs: an indentation was more often present in the hindlimbs than in the forelimbs. In $1.2 \%(n=15)$ of the joints, a fragment was present-always dorsal to, or partially superimposed on the sagittal ridge. The appearance of the sagittal ridge was evenly distributed over the five categories. The shape of the fragment varied from linear to oval, with a mean surface of $0.093 \mathrm{~cm}^{2}$ (from $0.011 \mathrm{~cm}^{2}$ to $0.472 \mathrm{~cm}^{2}$ ) ( Fig. 6A). In $1.7 \%(\mathrm{n}=20)$ of the joints, the presence of a fragment was suspected - always completely superimposed on the sagittal ridge ( Fig. 6B). In this case, the appearance of the sagittal ridge was mostly normal to irregular. One horse had a fragment in both forelimbs and one horse had a fragment in the left forelimb and hindlimb.

\section{Radiographic findings in area II}

The radiographic findings are summarised in > Table 1. In area II, $90.6 \%(\mathrm{n}=1091)$ of the joints had a normal appearance, $6.2 \%$ $(\mathrm{n}=75)$ were irregular, $2.9 \%(\mathrm{n}=35)$ had a depression or lucency at the sagittal ridge, and the sagittal ridge was flattened in $0.2 \%$ $(\mathrm{n}=3)$. No significant differences were found between left and right joints. A significant difference $(p<0.0001)$ in appearance of the distal part of the sagittal ridge was found between forelimbs and hindlimbs, with more depression or lucency present at the hindlimbs compared to the forelimbs. A fragment was present in $0.3 \%$ $(n=3)$ of the joints and was superimposed on the sagittal ridge. A depression was present at the level of the ridge in only one of the joints presenting a fragment. Although they were located more than $1 \mathrm{~mm}$ from the dorsoproximal aspect of the proximal phalanx, it was suspected that the other two fragments may have originated from the dorsoproximal aspect of the proximal phalanx. The fragments varied from linear $(\mathrm{n}=$ 2) to oval $(\mathrm{n}=1)$, with a mean surface of $0.078 \mathrm{~cm}^{2}$ (from $0.036 \mathrm{~cm}^{2}$ to $0.125 \mathrm{~cm}^{2}$ ) ( Fig. 7A). A fragment was suspected in 
five other cases; all were superimposed on the sagittal ridge and without a concurring lesion at the level of the sagittal ridge ( $>$ Fig. 7B).

\section{Other radiographic abnormalities}

An osseous remodelling or concavity at the dorso-distal aspect of the MCIII bone, just proximal to the sagittal ridge, was seen in 12 joints ( Fig. 8). This was a bilateral finding in five horses. Of these 12 joints, seven did not have any other radiographic abnormalities. In two joints (both forelimbs of the same horse) a notch was present in area I, one joint had a fragment at the dorsoproximal aspect of the proximal phalanx, and a mineralisation was detected at the level of the distal sesamoidean ligaments in 2 joints (forelimbs of the same horse).

Other abnormalities were osteochondral fragments in 33 joints $(2.6 \%)$ at the dorsoproximal aspect of the proximal phalanx ( 23 horses, 5 of which had two limbs affected).

\section{Discussion}

In our study, there was a lot of variation at the level of the dorso-proximal aspect of the sagittal ridge of the MCIII/MTIII. In a previous study performed on Thoroughbred horses, it was stated that these variations have no implications for future performance in sport (13). It is tempting to extrapolate

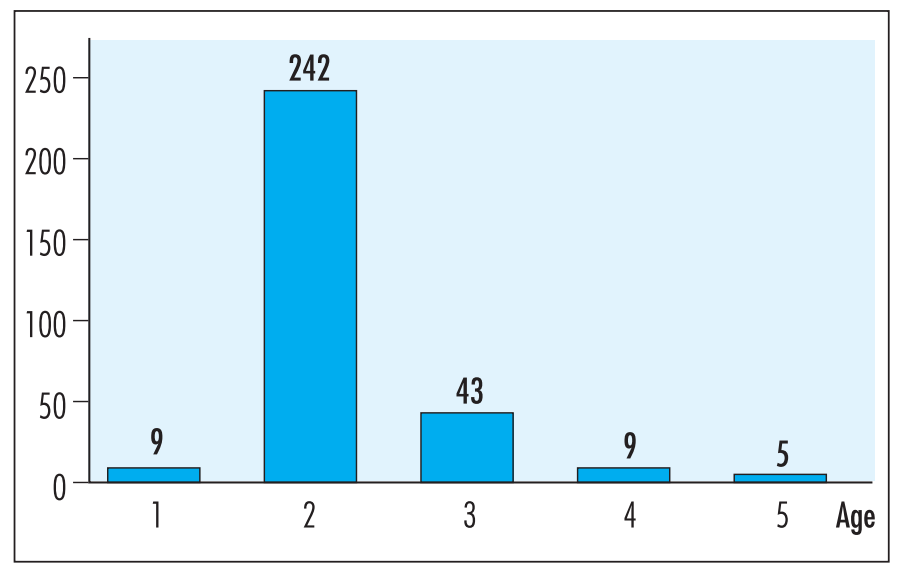

Fig. 5

Age (years) distribution of the horses. these conclusions. However, in contrast to the population of Thoroughbred horses used for racing, our population consisted of Warmblood horses used primarily for jumping or dressage (13). Compressive strains at the dorsal aspect of the MCIII/ MTIII have been shown to be more prevalent in racehorses working at high speed, whereas dressage or jumping horses - which usually work at a lower level of impact-have a different direction of strain, a longer sports career, and the possibility of other conformation shows as well (16-18).

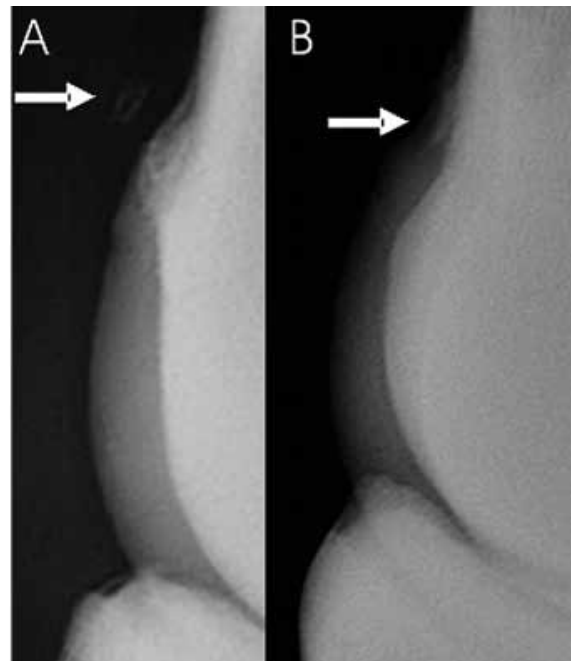

Fig. 6 Lateromedial radiograph of the fetlock showing the presence $A$ ) or the suspected presence $\mathbf{B}$ ) of a fragment in area I.

Table 1 Radiographic findings in metacarpo- and metatarsophalangeal joints in young Warmblood stallions.

\begin{tabular}{|c|c|c|c|c|c|c|c|c|c|c|c|}
\hline & \multirow[b]{2}{*}{ Category } & \multicolumn{2}{|c|}{ Left forelimb } & \multicolumn{2}{|c|}{ Right forelimb } & \multicolumn{2}{|c|}{ Left hindlimb } & \multicolumn{2}{|c|}{ Right hindlimb } & \multicolumn{2}{|l|}{ Total } \\
\hline & & $\mathbf{n}$ & $\%$ & n & $\%$ & n & $\%$ & $\mathbf{n}$ & $\%$ & $\mathrm{n}$ & $\%$ \\
\hline \multirow[t]{5}{*}{ Area 1} & Normal & 159 & 53.5 & 175 & 58.9 & 137 & 44.9 & 149 & 48.9 & 620 & 51.5 \\
\hline & Irregular & 61 & 20.5 & 60 & 20.2 & 54 & 17.7 & 57 & 18.7 & 232 & 19.3 \\
\hline & Notch & 28 & 9.4 & 24 & 8.1 & 30 & 9.8 & 25 & 8.2 & 107 & 8.9 \\
\hline & Lucency & 25 & 8.4 & 21 & 7.1 & 26 & 8.5 & 26 & 8.5 & 98 & 8.1 \\
\hline & Indentation & 24 & 8.1 & 17 & 5.7 & 58 & 19.0 & 48 & 15.7 & 147 & 12.2 \\
\hline \multirow[t]{4}{*}{ Area 2} & Normal & 286 & 96.4 & 282 & 94.9 & 260 & 85.2 & 263 & 86.2 & 1091 & 90.6 \\
\hline & Irregular & 6 & 2.0 & 12 & 4.0 & 29 & 9.5 & 28 & 9.2 & 75 & 6.2 \\
\hline & Depression / Lucency & 5 & 1.7 & 3 & 1.0 & 13 & 4.3 & 14 & 4.6 & 35 & 2.9 \\
\hline & Flattened & 0 & 0.0 & 0 & 0.0 & 3 & 1.0 & 0 & 0.0 & 3 & 0.2 \\
\hline Excessive obliquity & & 11 & 0.9 & 11 & 0.9 & 3 & 0.2 & 3 & 0.2 & 28 & 2.3 \\
\hline
\end{tabular}

Key: $\mathrm{n}=$ number. 
Fig. 7

Lateromedial radiograph of the fetlock showing the presence $A$ ) or the suspected presence $\mathbf{B}$ ) of a fragment in area II.

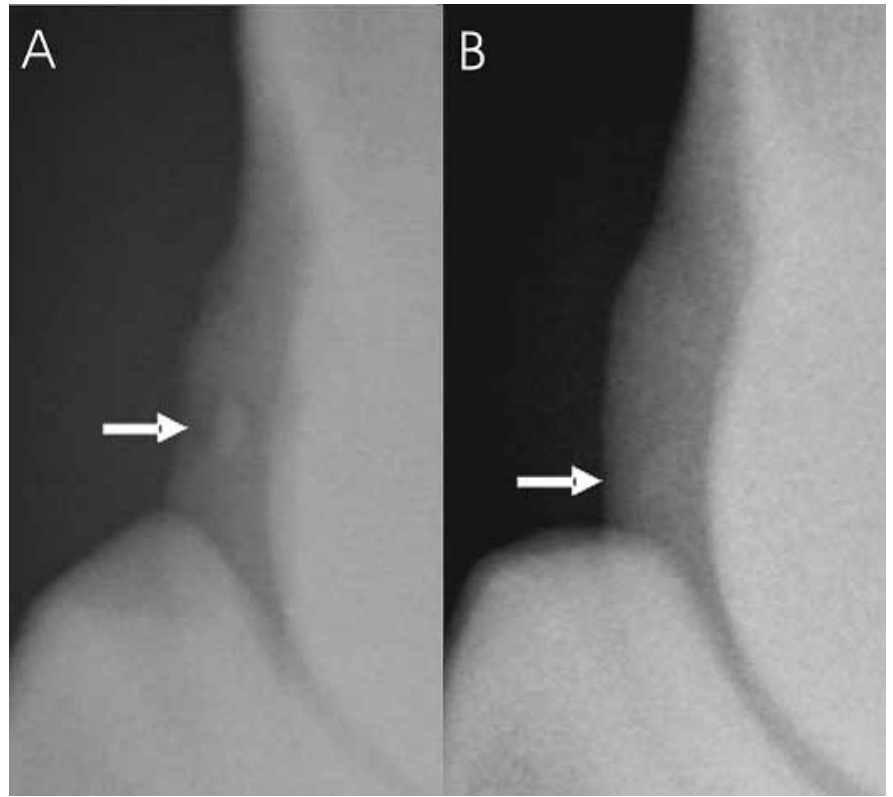

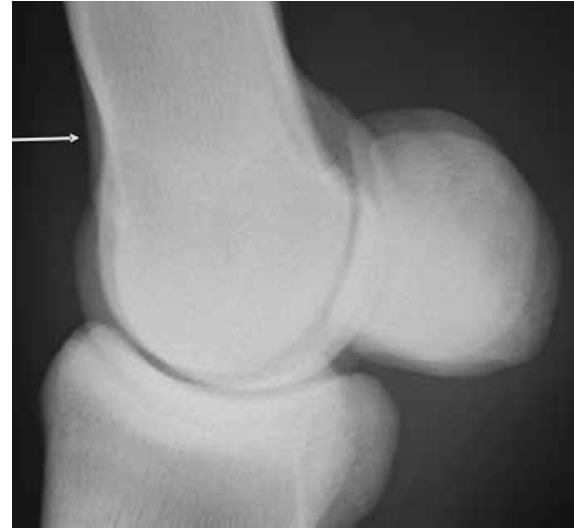

Fig. 8 Lateromedial radiograph of the fetlock showing an osseous concavity just proximal to the sagittal ridge.
In our study, $55 \%$ of the forelimbs and $46 \%$ of the hindlimbs showed a normal appearance of the dorso-proximal aspect of the sagittal ridge (area I), compared to $72 \%$ and $75 \%$, respectively, in a previous study of Thoroughbreds (9). Besides the difference in breed, this difference in percentages can be explained by the use of additional parameters (irregular and indentation) in the present study. If the metacarpo-/metatarso-phalangeal joints with an irregular outlining are considered to be normal, 77\% of the forelimbs and $65 \%$ of the hindlimbs fall into this category, which corresponds more closely to the previous study (9).

The presence of a notch was a bilateral finding in $23.3 \%$ of the forelimbs and in $27.7 \%$ of the hindlimbs of the horses in our study. This is in contrast to the result of a previous study in which this finding was bilateral in $65 \%$ of the horses (9). A lucency was present in 47 forelimbs and 53 hindlimbs, and was a bilateral finding in two horses and five horses, respectively. This is in agreement with the study of Kane et al. in which a lucency was most often a unilateral finding (9).

In the distal part of the sagittal ridge (area II), a difference between forelimbs and hindlimbs was present. For the forelimbs, our study found a higher percentage of normal joints compared to a previous study ( $95 \%$ in our study versus $78 \%$ in the previous study). However, for the hindlimbs, our study found a lower percentage of normal joints $(86 \%$ in our study versus $98 \%$ ) (9). In another population of Warmblood horses, $6.8 \%$ of the metacarpo-/ metatarso-phalangeal joints had an irregular surface of the sagittal ridge and one percent had a well-defined depression - much less than in our study (19). In addition, within the population from the other study, a fragment was present at the level of the sagittal ridge in $1.3 \%$ of the cases, compared to $0.3 \%$ in our study (19). A depression or lucency was significantly more frequent in this area in the hindlimbs. This finding is compatible with osteochondrosis and is in accordance with a previous study of a population of Standardbred trotters (20). In this area, the heritability of osteochondrosis has been demonstrated for both flattened bone contours and fragments (21).

A bone fragment was more frequently suspected than confirmed in both areas I and II. The difficulty in detecting a fragment can be due to poor fragment opacification, superimposition on other bony structures, or variation in soft tissues (22). Additional projections can partially address this problem, but they are not part of the standard radiographic protocol for the stallion selection at this institute. Ultrasonography has been shown to be superior to radiography for detecting and characterising dorsal bone fragments (23). However, performing an ultrasonographic examination in stallions for screening pur- poses is unrealistic. Other techniques such as arthroscopy, computed tomography or MRI may also enable detection of bone fragments (23-25). However, due to their cost and invasiveness, the use of these techniques is even less realistic in equine selection. As metacarpo- and metatarso-phalangeal joint fragments may have an influence on a horse's future lameness, it is important to detect them early (26). Radiography remains the diagnostic method of choice for the practitioner because of its low cost and high availability versus other imaging modalities (27).

In 23 horses $(7.2 \%)$, a fragment was present at the dorso-proximal aspect of the proximal phalanx. These fragments were found in 15 forelimbs and 18 hindlimbs. These results contrast with studies of Thoroughbreds and Standardbred trotters, in which most of the proximal phalanx fragments were located in the forelimbs $(20,28)$. However, in another study of Warmblood horses, the dorsal fragments were evenly distributed between the forelimbs and hindlimbs as in our study (26). Only one projection (latero-medial) was used in the present study, thus small fragments superimposed to the sagittal ridge may have been missed.

In 12 metacarpo-/metatarso-phalangeal joints, all of which were forelimbs, an osseous concavity was present just proximal to the sagittal ridge. Dabareiner et al detected 
this radiographic finding in $93 \%$ of a population of horses with joint synovial pad proliferation which was affecting the forelimbs (2). If we assume that this radiographic sign is indicative for synovial pad proliferation in our horses and compare the results, some differences are present. In our study, this radiographic abnormality was mainly a bilateral finding in 71\% (5 out of 7) of the horses, while in the study of Dabareiner et al, this was a unilateral finding in $79 \%$ (50 out of 63 ) of the horses (2). In the study of Dabareiner et al, all of the horses had lameness, joint effusion, or both signs associated with one or both metacarpophalangeal joints (2). These findings were not present in our study. On the other hand, this radiographic feature could also represent an anatomical variation in Warmblood horses instead of being a sign of synovial pad proliferation.

\section{References}

1. Denoix JM, Jacot S, Bousseau B, et al. Ultrasonographic anatomy of the dorsal and abaxial aspects of the equine fetlock. Equine Vet J 1996; 28: 54-62.

2. Dabareiner RM, White NA, Sullins KE. Metacarpophalangeal joint synovial pad fibrotic proliferation in 63 horses. Vet Surg 1996; 25: 199-206.

3. McIlwraith CW. Disease processes of synovial membrane, fibrous capsule, ligaments, and articular cartilage. In: Proceedings of the 47th Annual convention of the American Association of Equine Practitioners; 2001 November 24-28; San Diego, California, USA. pgs 142-156.

4. McIlwraith CW, Nixon AJ, Wright IA. Diagnostic and surgical arthroscopy of the metacarpophalangeal and metatarsophalangeal joints. In: McIlwrait CW, Nixon AJ, Wright IA, et al, editors. Diagnostic and surgical arthroscopy in the horse. Philadelphia: Elsevier; 2005. pgs. 129-196.
5. Vanderperren K, Saunders JH. Diagnostic imaging of the equine fetlock region using radiography and ultrasonography. Part 1: Soft tissues. Vet J 2009; 181: 111-122.

6. Vanderperren K, Saunders JH. Diagnostic imaging of the equine fetlock region using radiography and ultrasonography. Part 2: The bony disorders. Vet J 2009; 181: 123-136.

7. van Hoogmoed LM, Snyder JR, Thomas HL, et al. Retrospective evaluation of equine prepurchase examinations performed 1991-2000. Equine Vet J 2003; 35: 375-381.

8. McIlwraith CW, Kane AJ, Park RD. Changes on radiographs at thoroughbred yearling sales: prevalence and significance. Clin Tech Equine Pract 2003; 2: 329-338.

9. Kane AJ, Park RD, McIlwraith CW, et al. Radiographic changes in Thoroughbred yearlings. Part 1 : Prevalence at the time of the yearling sales. Equine Vet J 2003; 35: 354-365.

10. Becht JL, Park RD. A review of selected normal radiographic variations of the equine fetlock, carpus, tarsus and stifle. In: Proceedings of the 46th Annual Convention of the American Association of Equine Practitioners; 2000 November 26-29; San Antonio, Texas, USA. pgs. 362-364.

11. Bladon BM, Main JP. Clinical evidence in the evaluation of presale radiography: are we in a desert on a horse with no name? Equine Vet J 2003; 35 341-342.

12. Spike-Pierce DL, Bramlage LR. Correlation of racing performance with radiographic changes in the proximal sesamoid bones of 487 Thoroughbred yearlings. Equine Vet J 2003; 35: 350-353.

13. Kane AJ, McIlwraith CW, Park RD, et al. Radiographic changes in Thoroughbred yearlings. Part 2: Associations with racing performance. Equine Vet J 2003; 35: 366-374.

14. Cohen ND, Carter GK, Watkins JP, et al. Association of racing performance with specific abnormal radiographic findings in Thoroughbred yearlings sold in Texas. Equine Vet Sci 2006; 26: 462-474.

15. van Grevenhof EM, Ducro BJ, van Weeren PR, et al. Prevalence of various radiographic manifestations of osteochondrosis and their correlations between and within joints in Dutch Warmblood horses. Equine Vet J 2009; 41: 11-16.

16. Davies HMS, McCarthy RN, Jeffcott LB. Surface strain on the dorsal metacarpus of thoroughbreds at different speeds and gaits. Acta Anat 1993; 146: $148-153$.
17. Davies HMS, Merritt JS. Surface strains around the midshaft of the third metacarpal bone during turning. Equine Vet J 2004; 36: 689-692.

18. Bennell KL, Malcolm SA, Khan KM, et al. Bone mass and bone turnover in power athletes, endurance athletes, and controls: A 12-month longitudinal study. Bone 1997; 20: 477-484.

19. Verwilghen D, Serkeyn D, Pille F, et al. Prevalence of radiographic findings in candidate sires (2001-2008). Vlaams Dier Tijd 2009; 78: 419-428.

20. Grondahl AM. The incidence of bony fragments and osteochondrosis in the metacarpophalangeal and metatarsophalangeal joints of standard-bred Trotters - a radiographic study. Equine Vet Sci 1992; 12: $81-85$.

21. van Grevenhof EM, Schurink A, Ducro BJ, et al. Genetic variables of various manifestations of osteochondrosis and their correlations between and within joints in Dutch Warmblood horses. J Anim Sci 2009; 87: 1906-1912.

22. Widmer WR, Blevins WE. Radiographic evaluation of degenerative joint disease in horses - interpretive principles. Compend Contin Educ Pract Vet 1994; 16: 907-918.

23. Vanderperren K, Martens AM, Declercq J, et al. Comparison of ultrasonography versus radiography for the diagnosis of dorsal fragmentation of the metacarpophalangeal or metatarsophalangeal joint in horses. J Am Vet Med Assoc 2009; 235: 70-75.

24. Kawcak CE, McIlwraith CW, Norrdin RW, et al. Clinical effects of exercise on subchondral bone of carpal and metacarpophalangeal joints in horses. Am J Vet Res 2000; 61: 1252-1258.

25. Schneider RK, Sampson SS, Gavin PR. Magnetic resonance imaging evaluation of horses with lameness problems. In: Proceedings of the 51st Annual Convention of the American Association of Equine Practitioners; 2005 December 3-7, Seattle, Washington, USA. pgs. 21-34.

26. Declercq J, Martens A, Maes D, et al. Dorsoproximal proximal phalanx osteochondral fragmentation in 117 Warmblood horses. Vet Comp Orthop Traumatol 2009; 22: 1-6.

27. Horstmann W, Gerhards H, Hatami-Fardi M. Computed tomography in the navicular bone and in the distal interphalangeal joint of equine digit specimen compared to conventional radiography. Pferdeheilkunde 2003; 19: 511-519.

28. Kawcak CE, Mcilwraith CW. Proximodorsal first phalanx osteochondral chip fragmentation in 336 horses. Equine Vet J 1994; 26: 392-396. 